\title{
Los estudios de género y sexualidad en España a través de las revistas de geografía (1990-2014)
}

\author{
Xosé M. Santos \\ Universidade de Santiago de Compostela. Departamento de Xeografía \\ xosemanuel.santos@usc.es
}

\section{Resumen}

El objetivo de este artículo es evaluar la importancia que tiene la temática de género y sexualidad en la geografía española. Para ello, se analiza un total de diecinueve revistas españolas y diecisiete extranjeras correspondientes a los últimos veinticinco años. Esto supone un volumen superior a los 10.000 artículos. Consideramos que las revistas constituyen, en la actualidad, el mejor y más amplio medio de difusión de la investigación. Identificamos aspectos como la autoría, la universidad de adscripción, el contenido y el año de aparición, entre otros. Los resultados nos muestran la debilidad de los estudios sobre sexualidad y geografía. Por su parte, la perspectiva de género tiene una presencia relativamente temprana, aborda un abanico temático reducido, se concentra en unas pocas revistas y en un escaso número de autoras. Se destaca la internacionalización que va unida a la capacidad para abrir nuevas vías de investigación. Finalmente, se intenta poner en evidencia los motivos que generan esta situación tan precaria y particular de la geografía en España.

Palabras clave: geografía; género; sexualidad; revistas; España.

Resum. Els estudis de gènere $i$ sexualitat a Espanya a través de les revistes de geografia (19902014)

L'objectiu d'aquest article és avaluar la importància que té la temàtica de gènere i sexualitat a la geografia espanyola. Per fer-ho, s'analitzen un total de dinou revistes espanyoles i disset d'estrangeres corresponents als darrers vint-i-cinc anys. Això suposa un volum superior als 10.000 articles. Considerem que les revistes constitueixen, actualment, el millor mitjà de difusió de la recerca i el més ampli. Entre altres aspectes, n’identifiquem els corresponents a l'autoria, a la universitat d'adscripció, al contingut i a l'any d'aparició. Els resultats ens mostren la debilitat dels estudis sobre sexualitat i geografia. D'altra banda, la perspectiva de gènere hi té una presència relativament primerenca, aborda un ventall temàtic reduït, es concentra en poques revistes i en un nombre escàs d'autores. A l'article, s'hi destaca que la internacionalització va lligada a la capacitat d'obrir vies noves de recerca. Finalment, s'hi intenta posar en evidència els motius que generen aquesta situació tan precària i particular de la geografia a Espanya.

Paraules clau: geografia; gènere; sexualitat; revistes; Espanya. 
Résumé. Les études de genre et de sexualité en Espagne à travers les revues de géographie (1990-2014)

Cet article vise à évaluer l'importance du thème du genre et de la sexualité dans la géographie espagnole. Environ 10.000 articles correspondant à un total de 19 revues espagnoles et 17 étrangères ont été analysés (1900-2014). Nous considérons que les revues scientifiques constituent aujourd'hui le meilleur et le plus large moyen de diffusion de la recherche. Nou avons identifié certaines variables dans notre analyse, telles que l'auteur de l'article, l'université d'origine, le contenu et l'année de parution, entre autres. Les résultats montrent une absence évidente d'études sur la sexualité d'un point de vue géographique. D'autre part, la perspective de genre montre assez tôt sa présence, elle aborde un éventail réduit de thèmes et se concentre sur un petit nombre d'auteurs et de revues. En ce qui concerne les auteurs, il est important de souligner leur internationalisation qui est liée à la possibilité d'ouvrir de nouvelles lignes de recherche. Finalement, nous fournissons quelques raisons qui expliquent cette situation si précaire et particulière de la géographie espagnole.

Mots-clés: géographie; genre; sexualité; revues; Espagne.

\section{Abstract. Gender and Sexuality in Spain through Geographical Journals (1990-2014)}

This paper aims to assess the importance of gender and sexuality in Spanish academic geography. More than 10,000 articles published over the last 25 years in 19 Spanish and 17 international journals have been analysed. We believe that journals are currently the best and most comprehensive means for disseminating research. Items such as authorship, the author's academic affiliation, topic or date of publication, among others, have been identified. The results show that Spanish research on sexuality and geography has been rather scarce. Although the gender approach appeared relatively early on, only a narrow range of topics in a small number of journals have been dealt with by few, mostly female, authors. Nevertheless, these authors have succeeded in gaining international recognition and opening new research directions. Finally, the paper attempts to show the underlying reasons for the scarce development of gender and sexuality issues in the field of geography in Spain.

Keywords: geography; gender; sexuality; journals; Spain.

\section{Sumario}

1. Presentación. Género y sexualidad en la investigación geográfica española

\section{Metodología}

3. Resultados

4. Conclusiones

Referencias bibliográficas 


\section{Presentación. Género y sexualidad en la investigación geográfica española}

El objetivo de este artículo es analizar la relevancia que tienen en España los estudios de género y sexualidad y su reflejo en las revistas de geografía. Para ello trabajamos siguiendo una doble vía. Por un lado, analizamos las principales publicaciones periódicas de nuestra disciplina que se editan en España. Por otro, nos fijamos en algunas de las revistas más representativas del panorama internacional con el fin de observar la presencia española en las mismas y evidenciar el protagonismo y la visibilidad que, en conjunto, tienen género y sexualidad en ellas.

La geografía del género y de las sexualidades forma parte del eco académico que tuvieron los movimientos sociales vinculados con el feminismo y con la liberación gay y lesbiana. En el primero de los casos, nos situamos en la década de 1970 y, en el segundo, unos años más tarde. Es el ámbito anglosajón el primero que recoge estas demandas y las transforma en análisis para dar solución a los múltiples problemas y conflictos que surgen en torno a las desiguales relaciones que manifiestan el género y la sexualidad con respecto al espacio.

Zelinsky (1973) es el primero que habla abiertamente de la escasa representación que tienen las mujeres en la geografía. Algunos artículos posteriores, como los de Palm y Pred (1976) (apud Garcia Ramon, 1989), no hacen más que constatar la evidencia de las desigualdades de género. Pero sin duda fue la creación, en 1982, de un grupo de trabajo sobre mujeres en el Instituto Británico de Geografía la que recogió una creciente necesidad e impulsó fuertemente la perspectiva de género y los estudios feministas dentro de la geografía. A partir de aquel momento, se multiplicaron las investigaciones y las publicaciones académicas.

Con relativamente poco retraso, esta influencia anglosajona también llega a España y ve la luz en 1982, gracias a un artículo de García Ballesteros (Garcia Ramon, 1989). En los años siguientes, otras autoras, fundamentalmente de Madrid y Barcelona, irán incorporando nuevas aportaciones, si bien el ritmo de las mismas es bastante irregular. En cualquier caso, lo relevante es que se van marcando líneas de investigación que se vinculan a ciertas profesoras y a centros muy concretos. Su visibilidad se incrementará claramente en los años siguientes.

Durante la etapa que se prolonga hasta la entrada del nuevo siglo, la geografía feminista en España muestra tres características relevantes, según destaca Ortega Valcárcel (2000): se dedican a ella un número reducido de profesionales, es desarrollada fundamentalmente por mujeres y se centra de manera muy particular en los ámbitos rural y agrario. Ese mismo autor se refiere al dinamismo de esta parte de la geografía humana, así como a la relevancia que adquiere la producción teórico-metodológica, lo que la diferencia con respecto al conjunto de la geografía española.

En cuanto a la geografía de las sexualidades, su desarrollo es más tardío, tanto en el mundo anglosajón como en el español. Las primeras referencias en 
Estados Unidos se pueden establecer en Lauria y Knopp (1985), para adquirir posteriormente más relevancia en la década de 1990. Sin embargo, hay otros antecedentes que merecen ser citados, como el capítulo que Castells (1983) le dedica a la construcción social del barrio del Castro en San Francisco. También desde la sociología, Ettorre (1978) analiza el gueto lésbico de Lambeth en Londres a partir de la ocupación de viviendas vacías. En España, la referencia inicial en revistas geográficas se retrasa hasta el año 2000, si bien existen algunas aportaciones anteriores en publicaciones de carácter local o poco conocidas para la geografía española (Santos, 1996 y 1998). De todas formas, la notoriedad de estas publicaciones es muy reducida, característica que se mantendrá en los años siguientes.

Nos parece importante reflexionar sobre las causas que dan lugar a esta situación de escasez alarmante en los estudios de geografía y sexualidad. Entendemos que, probablemente, existan múltiples motivos que entrelacen razones de tipo académico con otras de carácter personal. Por un lado, creemos que existe un cierto temor a que las personas autoras de estos trabajos sean identificadas con aquellas orientaciones sexuales sobre las que investigan, fundamentalmente con la homosexualidad. Por otro lado, pensamos que existe una baja consideración de la sexualidad como objeto de estudio geográfico, bien por ser visto como un tema poco serio o, simplemente, por desconocimiento o incapacidad para incorporar el enfoque territorial a la sexualidad. Es bueno recordar las duras críticas recibidas por Knopp cuando escribió el artículo titulado «Social consequences of homosexuality» para la Geographical Magazine (1990). Por primera vez, una publicación geográfica muy ampliamente distribuida en los Estados Unidos abordaba este tema, lo que valió, al autor y a la propia revista, reprimendas por parte de algunos lectores sobre el rumbo tomado. En este mismo sentido, citamos a Valentine, quien, en un apasionante artículo (1998), se introduce en su propia biografía para explicar las enormes complicaciones derivadas de su investigación.

Dejando ahora de lado las revistas, que analizaremos en el apartado correspondiente y que forman el núcleo de la presente investigación, la sexualidad apenas ha sido objeto de estudio en la geografía española. Resulta extremadamente complicado llegar a la literatura gris que se produce a partir de proyectos o de trabajos académicos. Sin embargo, hemos conseguido localizar algunas referencias en departamentos de Madrid, Zaragoza o Salamanca. Entre ellas, podemos citar a Silva (2006), con un trabajo de investigación tutelado bajo la orientación de Vicente Mosquete. Muchas veces estamos ante estudios iniciáticos vinculados a la antigua licenciatura, al grado o a los másteres, no siempre exclusivamente de geografía, que después no tienen continuidad académica.

Hemos localizado también algún autor escribiendo sobre geografía y sexualidad en revistas que no son estrictamente de esta disciplina y que se vinculan a áreas de gran transversalidad, como el turismo. Es el caso, por ejemplo, de Dóniz (2013) o de Prat (2014), entre otros. También manuales y libros de geografía incorporan en algunos casos el tema de la sexualidad. Destaca la obra Las otras geografias, coordinada por Nogué y Romero (2006), cuya cuarta parte 
está dedicada al cuerpo y contiene tres capítulos (Bru, Prats y Santos). Todavía menos visibilidad tiene la geografía de la sexualidad en libros de otras materias o de clara transversalidad (Santos, 2006).

Finalmente, haremos una breve referencia a las reuniones científicas. Merecen una especial atención los congresos europeos de geografía de las sexualidades, cuyas dos ediciones tuvieron lugar en Bélgica (2011) y Portugal (2013). El de 2015 está programado en la ciudad italiana de Roma. En el caso de Lisboa, en el programa del evento, se ve que la geografía española tuvo una presencia casi testimonial, a pesar de encontrarse cerca de la ciudad organizadora. Según constaba en el citado programa, la representación de personas procedentes de España fue de once, la mayor parte de ellas vinculadas con otras disciplinas ${ }^{1}$. En concreto, y por lo que respecta a la geografía, hemos encontrado una persona en el comité científico y otra con participación activa en las diferentes sesiones.

Otro evento en el que estuvieron presentes los estudios de sexualidad como objeto de análisis fue el II Seminario Latinoamericano de Geografía de Género y Sexualidad ${ }^{2}$, celebrado en Rondônia (Brasil) en 2014, con una pequeña representación del Estado español ${ }^{3}$. Por último, una breve referencia al XIV Coloquio Ibérico de Geografía, celebrado en Guimarães en 2014, que por primera vez incluyó un eje temático en torno a la sexualidad. De las doce comunicaciones presentadas, solo dos tenían autoría española, lo que evidencia una vez más la escasa relevancia de los estudios geográficos sobre sexualidad.

En cuanto al género, el papel que tiene en la geografía española es significativamente mayor. En su obra de referencia Los horizontes de la geografía (2000), Ortega Valcárcel dedica un capítulo entero al que señala, entre otras cosas, que es un fenómeno reciente, si bien demuestra gran dinamismo en ciertos contextos como el anglosajón. La visibilidad que le da Ortega Valcárcel se repite en el informe La investigación geográfica en España (1990-2012) (Lasanta y Martín Vide, 2013), que incluye a la geografía del género como una subdisciplina. Debido a que el período temporal de dicho informe coincide con el de este artículo, utilizaremos algunos de sus datos para abordar este apartado inicial.

Pero antes parece conveniente hacer una breve referencia a los trabajos pioneros anteriores al año 1990. Como ya comentamos, un artículo publicado por García Ballesteros en 1982 es considerado el que abre el camino a la geografía del género en España, siguiendo a continuación otros de autoras como Sabaté (1984a y 1984b) o Garcia Ramon (1985a). Estos trabajos ven la luz con no demasiado retraso en relación con lo que se había estado haciendo en el mundo

1. Se hizo una búsqueda por Internet de todas las personas vinculadas a alguna universidad española, independientemente de la nacionalidad de dicha persona. Fueron identificadas nueve de esas once personas y solo una fue reconocida como geógrafa.

2. El primer seminario se celebró en Rio de Janeiro en 2011, si bien era exclusivamente de género.

3. En realidad, por lo que a la parte de sexualidad se refiere, son las mismas personas que acudieron al congreso de Lisboa. 
anglosajón, debido a los contactos internacionales establecidos entre investigadoras. En todo caso, se comienza a visibilizar el papel de la mujer en relación con el uso del espacio aportando novedosos enfoques teóricos. También es importante destacar en este proceso el papel protagonista de algunas revistas ampliamente reconocidas en el ámbito académico de la geografía española, como Anales de Geografía de la Universidad Complutense o Documents d'Anàlisi Geogràfica $(D A G)$.

Además de las españolas, también en esta primera etapa encontramos algún artículo en revistas internacionales, como el de Garcia Ramon et al. (1988) en la prestigiosa The Professional Geographer, bajo el título "Woman and Geography in Spanish Universities», o el trabajo de Garcia Ramon y Cànoves (1988) en Sociologia Ruralis sobre el papel de la mujer en la agricultura familiar catalana. Esta salida al exterior va acompañada de un refuerzo interior con la publicación de la obra Teoría y método en la geografía humana anglosajona (Garcia Ramon, 1985b), puesta en el mercado por una editorial de amplia difusión. Aunque dicho libro aborda una multiplicidad de temas, encontramos, por ejemplo, una traducción de un artículo de Bowlby et al. (1982) aparecido originariamente en Area bajo el título "Feminism and Geography».

Por fin, en 1989, encontramos un primer monográfico dedicado a género en una revista española: $D A G$. Su origen está en el seminario Agricultura, Género y Espacio, organizado dos años antes por el Departamento de Geografía de la Universidad Autónoma de Barcelona en colaboración con el Instituto de la Mujer y al que asistieron algunas de las pioneras anglosajonas de esta subdisciplina (Cánoves y Solsona, 1989). En ese volumen de $D A G$, ya se advierte la importancia que el mundo rural va a adquirir en los estudios de género en España, así como también algunas de las autoras más representativas en los años a seguir.

Llegamos así a 1990, año a partir del cual el informe elaborado por la Asociación de Geógrafos Españoles (AGE) nos servirá de guía para evaluar la relevancia de los estudios de género y sexualidad en los diferentes ámbitos de la investigación geográfica en España, excluidas las revistas para las que haremos nuestro propio análisis. En lo que se refiere a los proyectos de I+D, el informe elaborado por Esparcia (2013) se centra únicamente en el Plan Nacional, que es el más clásico de los programas de financiación de la I+D+i en España. En este contexto, los proyectos sobre género ocupan una posición muy residual y ligada casi en exclusiva con la Universidad Autónoma de Barcelona. De los 272 analizados, solo 3, es decir el 1,1\%, estarían relacionados con la geografía social y del género. El autor del texto destaca el escaso peso de esta temática, especialmente si se contempla, según él, la relativa importancia que tiene en España la geografía del género.

En el capítulo dedicado a los grupos de investigación, Olcina y Lois (2013) citan únicamente a dos que están orientados a los estudios de género, hecho que evidencia una significativa debilidad, considerando el papel crecientemente importante que tienen los grupos. Con todo, también conviene destacar que uno de ellos fue creado en fechas tan tempranas como el año 1987 (Cànoves, 1999). 
Conforme el informe elaborado por la AGE incorpora nuevos aspectos de la investigación, se hace más evidente el escaso peso que muestra la geografía del género. Así, en cuanto a las monografías, aparecen citadas únicamente once, que es una cifra muy baja en relación con el volumen total de libros publicados. Este mismo hecho se repite en el ámbito de las tesis doctorales, de capítulos de libros o en el número de comunicaciones presentadas en los congresos generales de geografía, en donde el porcentaje no llega al $1 \%$.

Por lo tanto, se puede concluir que la investigación sobre la geografía del género es, en España, bastante pobre en términos cuantitativos, a pesar de que se puede considerar que su abordaje se produce en fechas bastante tempranas.

A continuación, pasaremos a centrar nuestra atención en las revistas, con el objetivo de analizar la importancia que tienen las temáticas de género y sexualidad. El motivo de fijar el foco en este medio de difusión es porque creemos que, en estos momentos, es el más popular y el más valorado para la publicación de la investigación. Además de su número, su versatilidad y la facilidad de acceder a sus contenidos, destaca el hecho del papel adquirido en la evaluación de la calidad investigadora a través del sistema de sexenios, que se ha convertido en una pieza fundamental para el reconocimiento académico, lo cual ha generado una gran demanda y expectación. Por último, y como ya señalaban Garcia Ramon y Caballé (1998), «academic journals are more sensitive to the emergence of new topics» (p. 210).

\section{Metodología}

Como se acaba de decir, el objetivo de este artículo es el de analizar la importancia de la investigación sobre geografía del género y de las sexualidades en las revistas geográficas. El período temporal está situado entre los años 1990 y $2014^{4}$. Desde el punto de vista metodológico, la principal dificultad deriva de la comparación entre las revistas editadas en España y las que lo son en el extranjero, debido a que la información que se busca en unas y otras no es exactamente la misma. En el caso de las revistas españolas, nos centramos en los artículos cuya temática es el género y la sexualidad, teniendo en cuenta, además, otras variables, como ya veremos. Sin embargo, en las publicaciones foráneas, ponemos el foco fundamentalmente en la autoría, evidentemente vinculada con una temática. Pero, a diferencia del caso español, no consideramos la totalidad de los artículos que aparecen sobre género y sexualidad.

El período temporal escogido va entre 1990 y 2014. Se necesita justificar la primera de las fechas, que no es utilizada meramente por tratarse del inicio de una nueva década. Desde el punto de vista académico, el motivo está en la

4. El período de redacción de este artículo se cerró el 28 de febrero de 2015, por lo que no todas las revistas tenían en dicha fecha el volumen de 2014 publicado al completo. En concreto, quedaron por completar, de manera parcial o total, las siguientes revistas: Polígonos, Cuadernos de Geografia de la Universitat de València, Geographicalia y Boletín de la Real Sociedad Geográfica. 
publicación en 1989 de un primer monográfico en la revista $D A G$ dedicado al género. A partir de ese momento, esta subdisciplina deja de ser una anécdota de algunas geógrafas y entra de lleno en el campo de la investigación. Adquiere, por lo tanto, visibilidad y relevancia en España a través de una revista de amplia difusión, de unas autoras reconocidas y de un campo de trabajo bien implantado en otros países de referencia.

La selección de las revistas españolas fue realizada utilizando el índice $\mathrm{H}$ de las revistas españolas de geografía para 2012, que elabora el grupo de investigación EC3 de la Universidad de Granada ${ }^{5}$, si bien no nos interesaba el posicionamiento de cada una de ellas, sino solo su relación. La práctica totalidad es bien conocida en la geografía española, aunque no todas fueron tomadas en consideración. De las 47 que recoge el índice, se excluyeron aquellas que tenían una serie muy reducida y que ya no se publican. Por el contrario, han sido incluidas otras recientemente desaparecidas pero de larga trayectoria. No se tuvieron en cuenta revistas vinculadas a facultades o a instituciones en las que la geografía tiene una representación escasa. Del mismo modo, tampoco fueron analizadas revistas de temática muy específica. Por citar algunos ejemplos de estas casuísticas excluyentes, tenemos: Xeográfica, Revista de Estudios Andaluces, Cuadernos de Turismo o Ciudad y Territorio, entre otras.

Finalmente, ese total de 47 revistas fue reducido a $19^{6}$, la mayor parte de las cuales están vinculadas a los diferentes departamentos de Geografía de las universidades españolas, aunque también a otros organismos e instituciones, como el CSIC, la AGE o la Real Sociedad Geográfica (RSG). Si consideramos el período de veinticinco años y las diecinueve revistas, obtenemos una cifra algo superior a los 6.000 artículos, si bien es cierto que ha habido cambios importantes a lo largo del tiempo, de tal manera que se ha evolucionado hacia un incremento en el número de artículos y de volúmenes ${ }^{7}$. Ante la imposibilidad de leer todos los artículos, se precisaron modos alternativos para una aproximación a su contenido. Por un lado, estaba la posibilidad de buscar a través de las palabras clave, método que permite utilizar la mayor parte de las revistas en formato digital. Sin embargo, fue desechado después de comprobar la multiplicidad de vocablos utilizados y, sobre todo, porque no siempre había

5. Véase <http://ec3.ugr.es/publicaciones/indiceh_ciencias_sociales.pdf $>$.

6. En realidad, fueron dieciocho las revistas incluidas en ese índice más Polígonos, editada por los departamentos de Geografía de León, Valladolid y Salamanca. Las otras dieciocho revistas analizadas son: Scripta Nova (Geocritica), Boletin de la Asociación de Geógrafos Españoles (BAGE), Investigaciones Geográficas, Ería, Papeles de Geografía, Anales de Geografía de la Universidad Complutense, Documents d'Anàlisi Geogràfica, Cuadernos Geográficos de la Universidad de Granada, Estudios Geográficos, Treballs de la Societat Catalana de Geografia, Cuadernos de Investigación Geográfica, Cuadernos de Geografía de la Universitat de València, Lurralde, Boletín de la Real Sociedad Geográfica, Didáctica Geográfica, Serie Geográfica y Espacio, Geographicalia, Tiempo y Forma (Geografia).

7. Por ejemplo, el Boletín de la $A G E$ tenía, en 1990, dos números y un total de diez artículos, mientras que en 2014 eran tres números y más de cincuenta artículos. De hecho, en general, para el período más reciente, contabilizamos unos cien artículos más por año con respecto a 1990. 
una correspondencia clara entre palabras clave y contenido. Por otro lado, estaba el recurso al título del artículo, que fue la vía seguida, al entender que da pistas muy aproximadas del contenido ${ }^{8}$. Además, en caso de dudas, una lectura del mismo servía para aclararlas. Con todo, reconocemos que es probable que se nos escapase alguno, aunque creemos que, en cualquier caso, serían pocos y que apenas variarían los resultados.

Los datos que analizamos fueron los siguientes: nombre de la revista, año de publicación, autoría y universidad o institución a la que se adscribe esa autoría. Hemos hecho un intento de organizar los trabajos en base a la temática abordada, además de tratar de delimitar el ámbito territorial de la investigación. Ambas cuestiones, sobre todo la primera, no han resultado fáciles. La nacionalidad y el campo disciplinario de los investigadores y las investigadoras nos han interesado, aunque la mayoría de los artículos se corresponden con personas españolas y de geografía. Por más que estos últimos datos no eran relevantes, a veces resultaban difíciles de averiguar. En ocasiones, porque no se hace referencia a ninguna adscripción departamental concreta; también porque el nombre no siempre denota nacionalidad, particularmente si consideramos América Latina. De la misma manera, también existen investigadoras e investigadores extranjeros en universidades españolas y viceversa. Finalmente, el idioma del texto ha sido otro punto de interés secundario.

Como complemento a las revistas españolas, nos hemos interesado también por publicaciones periódicas extranjeras. Este hecho, como ya hemos señalado, nos generó ciertas dificultades. Una de ellas consistió en su elevado número existente, que nos obligó a realizar una selección no exenta de cierta subjetividad. Como criterio general, hemos recurrido a la clasificación JCR (2013), por ser tal vez la más restrictiva y, supuestamente, la más rigurosa. De las 76 revistas indexadas ${ }^{9}$, hemos escogido $17^{10}$, procurando incluir desde las más generalistas hasta otras con marcado carácter temático dentro de la geografía humana. Entre las primeras, están algunas de las más reconocidas en nuestro campo disciplinario, como Progress in Human Geography, Transactions of the $I B G$ o Geoforum. En referencia a las temáticas, hemos optado por aquellas que, a nuestro juicio, abarcan los macroejes que conforman la geografía humana.

8. La mayoría de las revistas fueron consultadas a través de Internet, gracias a que casi todas están digitalizadas. De todas formas, algunas fueron revisadas en papel. En toda esta serie de veinticinco años, hubo pequeñas incidencias, como algún número perdido imposible de localizar o el caso de alguna revista que empieza su recorrido después de 1990 o que cesa su actividad antes de 2014 .

9. De esas 76 revistas, dos son españolas: BAGE y Scripta Nova.

10. Las diecisiete revistas seleccionadas son: Progress in Human Geography, Transactions of the IBG, Antipode, Geoforum, Social and Cultural Geography, Environment and Planning D: Society and Space, Population, Space and Place, Environment and Planning A, International Journal of Urban and Regional Research, Area, The Professional Geographer, Sociologia Ruralis, Children's Geographies, Gender, Place and Culture, Tijdschrift voor Economische en Sociale Geografie y Journal of Geography in Higher Education. Además de todas las citadas, referenciadas en JCR, incluimos una más, creada en 2008, que, por sus características, nos parecía interesante para nuestro objeto de estudio: Emotion, Space and Society. 
De esta forma, se incorporaron, entre otras, Social and Cultural Geography, la revista Population, Space and Place, el Journal of Geography in Higher Education o Sociologia Ruralis. Finalmente, hay ciertas publicaciones en las que el género y la sexualidad representan el objeto de las mismas o al menos han demostrado una tendencia muy clara hacia esos contenidos, como Gender, Place and Culture, Children's Geographies o Emotion, Space and Society.

Para terminar, es conveniente decir que este no es un estudio bibliométrico en sentido estricto y cuyo objetivo sería "estudiar, contar, clasificar y evaluar la producción y el consumo de información científica mediante métodos cuantitativos y tratamiento estadístico» (Sanz y Martín, 1997: 43). Las características de este trabajo se acercan más a otros como el de Garcia Ramon y Caballé (1998), que tenían como meta principal evaluar el impacto de la perspectiva de género en la geografía académica, centrándose fundamentalmente en las revistas. Para ello, es suficiente el análisis de perfil como hacen Corral y Cànoves (2013) en relación con el turismo.

\section{Resultados}

Si empezamos por las revistas españolas, excluidas las notas, las reseñas y los comentarios, en el período 1990-2014, hemos encontrado 188 artículos que tratan, de una u otra forma, el tema del género y/o la sexualidad ${ }^{11}$. Aunque no tenemos una cifra exacta de artículos revisados, considerando los algo más de 6.000 que estimamos para las revistas españolas, estaríamos hablando de aproximadamente un 3\%, volumen que, en su conjunto, nos parece bastante bajo. Si desagregamos en las dos temáticas que estamos analizando, los 9 artículos sobre sexualidad revelan unas carencias muy significativas en este ámbito.

\subsection{Los trabajos sobre sexualidad}

La tabla 1 nos permite visualizar algunas de las características de estas investigaciones. Recordemos que los primeros artículos tardan bastante en aparecer. De hecho, no encontramos alguno hasta 1999. A partir de ese momento, tampoco se desarrolla ninguna línea de investigación y solo aparecen publicados algunos trabajos dispersos, sin que destaque ningún autor o autora.

Como se puede observar, hay una concentración importante en algunos apartados. Nos referimos al año 2007, a dos revistas, a la autoría masculina y a la temática de la homosexualidad. No es relevante, sin embargo, ninguna universidad en particular, así como ningún autor o autora, por lo que existe, como se ha dicho anteriormente, una notable dispersión. Si afinamos un poco

11. No vamos a establecer ninguna comparación con el estudio realizado por la AGE sobre la investigación geográfica en España entre 1990 y 2012 (Lasanta y Martín, 2013), porque la metodología utilizada es diferente. Ver el capítulo de ese informe elaborado por Martín et al. (2013: 235-248). 
Tabla 1. Características principales de los estudios sobre sexualidad en las revistas geográficas españolas

\begin{tabular}{ll}
\hline Año & $2007(44,4 \%)$ \\
Revista & DAG $(44,4 \%)$, Anales Complutense $(22,2 \%)$ \\
Autoría & Hombres $(77,7 \%)$ \\
Temática & Homosexualidad $(66,6 \%)$ \\
\hline
\end{tabular}

Fuente: revistas de geografía españolas.

más, es cierto que la Universidad Complutense de Madrid es el origen de dos artículos, aunque uno de ellos parte del ámbito disciplinario de la filología. También es relevante que un tercio de los artículos estén vinculados a centros de investigación extranjeros, en concreto de Brasil, Chile y Canadá.

Si ponemos el foco en las revistas internacionales, la realidad es todavía más contundente, puesto que no hemos encontrado ningún artículo publicado en el período analizado. Tan solo Children's Geographies nos avanza un trabajo de Rodó, cuya versión en línea de 2013 no había salido publicada en papel en el año 2014. Esta autora, vinculada a la UAB, desarrolla una línea de investigación que está claramente orientada hacia la sexualidad y el feminismo, mostrando una evidente internacionalización, si bien, si se incluye el año 2014, sus referencias en las revistas analizadas se limitan a la citada y a otro artículo de género aparecido en Gender, Place and Culture, también en 2014.

\subsection{Las investigaciones sobre género en España}

Frente a la casi irrelevancia de la sexualidad en los estudios geográficos publicados en revistas españolas o extranjeras, observamos que, en la cuestión de género, el panorama es mucho más optimista, aunque sin olvidar que, en el conjunto del medio de difusión analizado, su representación continúa siendo mejorable. Recordemos que estamos haciendo referencia, para el caso de España, a 179 artículos a lo largo de 25 años, lo que, llevado a una simple media, nos daría un valor de 7,16 artículos por año. Y una vez más debemos de tener en cuenta que son 19 las revistas consideradas.

Una mirada a la tabla nos permite ver algunas evidencias bastante claras sobre la evolución en la aparición de artículos. Observamos cuatro picos (en realidad, cinco, al solaparse dos de ellos), que se corresponden con los monográficos ${ }^{12}$ editados y que generan importantes modificaciones. Si prescindimos de las distorsiones que provocan, es perceptible una línea menos sinuosa que se mueve entre dos y siete artículos. En todo caso, se advierte un mayor dinamismo en la década de 1990, que tiene, además, tres de esos cinco monográficos. Los dos números especiales del siguiente decenio son de tamaño

12. La revista $D A G$ tiene tres monográficos (1995, 1999 y 2007); Cuadernos de Geografía de la Universitat de València, uno, editado en 1998; finalmente, en 2002, Scripta Nova publica, en un número extraordinario, las actas del IV Coloquio Internacional de Geocrítica, que incluye ocho trabajos del área temática Trabajo y Género. 


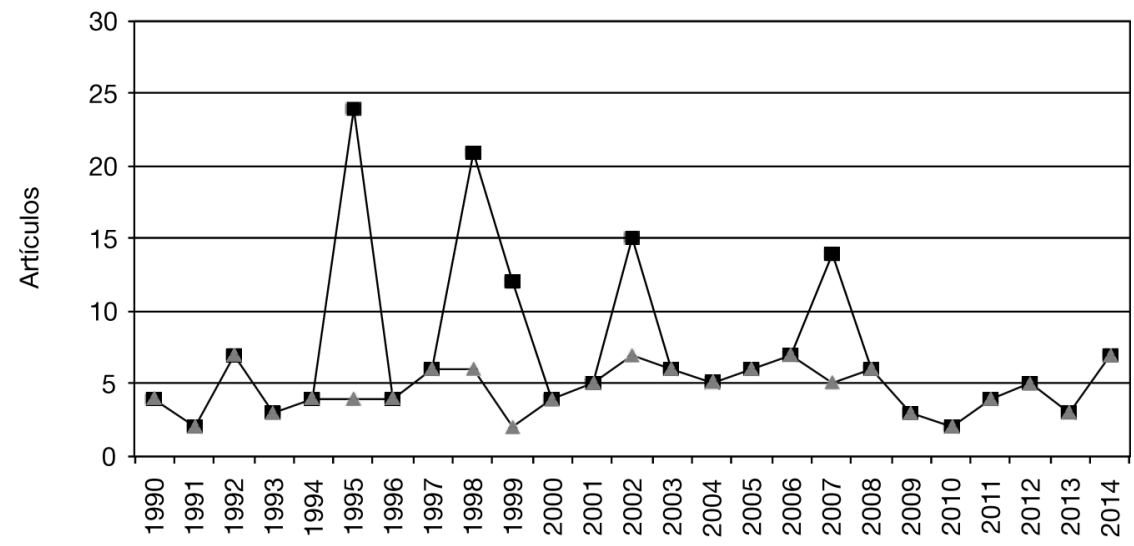

Años

- Incluidos monográficos

\section{- Excluidos monográficos}

Gráfico 1. Artículos sobre género publicados en las revistas de geografía españolas.

Fuente: revistas de geografía españolas.

más reducido. Sin embargo, también es cierto que, entre los años 2000 y 2009, es cuando se aprecia una media más elevada, siempre y cuando se elimine el efecto de los monográficos. Finalmente, entre 2010 y 2014, se mantienen unos niveles ligeramente inferiores a la etapa precedente, con un repunte en 2014.

Una lectura como la realizada en el párrafo anterior se quedaría corta si no consideráramos la evolución física de las revistas analizadas. Es cierto que hay algunas que han desaparecido en los años más recientes, pero eso no compensa el fuerte crecimiento en artículos que han experimentado la mayor parte de las mismas. De hecho, si analizamos los años inicial y final de este trabajo, observamos que, en 1990, todas las publicaciones vistas tenían algo menos de 250 artículos por año y, en $2014^{13}$, eran casi 100 más. Este progresivo salto cuantitativo tan importante hace que, en términos relativos, no sea lo mismo los siete artículos de 1992 que los de 2014.

La variable que representa a las revistas nos deja algún resultado sorprendente. Por ejemplo: existe un 26,3\% de revistas que no tienen ningún artículo sobre género. Este hecho resulta más llamativo si consideramos que, en algunos casos, son publicaciones con una larga trayectoria e incluso con prestigio en la geografía española. En el lado opuesto, podemos afirmar que hay una notable concentración en algunas revistas, como $D A G$, que acumula el $41,3 \%$ de ellos; Cuadernos de Geografía de la Universitat de València, con el 13,9\%;

13. En el caso de aquellas revistas no publicadas total o parcialmente en 2014, se consideró y/o se completó con el último año disponible. 
Scripta Nova, con el 8,9\%, o Treballs de la Societat Catalana de Geografia, con el $6,7 \%$. Entre estas cuatro reúnen el $70,8 \%$ del total, dejando poco margen para el resto.

Sin embargo, dando un paso más y eliminando la distorsión de los números monográficos, tenemos otro dato bien interesante: la única revista que mantiene una continuidad en el tiempo es $D A G$, que, en el total de la serie, solo en dos años no tiene ningún artículo sobre género; le seguirían Treballs de la Societat Catalana de Geografia y Cuadernos de Geografía de la Universitat de València, con artículos en diez y nueve años, respectivamente. Por el contrario, Scripta Nova apenas tiene nada publicado fuera del monográfico incluido en el Congreso de Geocrítica.

Otro hecho que llama poderosamente la atención es la escasa importancia que tiene la temática del género en la revista que pasa por ser la más representativa de la geografía española: el $B A G E$. En total, son únicamente diez artículos, de los que tres se corresponden con el año 2014. Si bien el primero aparece en fechas relativamente tempranas, los demás (de hecho, nueve) son de 2006 en adelante. Recodemos que el $B A G E$ ha tenido, en términos cuantitativos, un crecimiento espectacular, puesto que ha pasado de ser bimestral a ser cuatrimestral y ha multiplicado el número de trabajos por volumen. Así, entre 2006 y 2014, se editaron algo más de 400 artículos, lo que significa que el género representó un escaso $2 \%$, lo cual da lugar a una situación poco acorde con lo que cabría esperar de una publicación con aspiraciones de liderazgo.

Destaca también el hecho de que todas las revistas en las que los estudios de género tienen una cierta impronta están editadas en Cataluña o, en todo caso, en el ámbito de la lengua catalana, si incluimos Cuadernos de Geografía de la Universitat de València. Esto sirve para entender la relevancia que adquiere el idioma catalán, que representa cerca del $40 \%$ del total, frente al $50 \%$ del castellano. El resto está escrito en otras lenguas, como el inglés o el portugués.

La importancia que adquiere Cataluña, tanto en el idioma como en los lugares de edición de las revistas, se manifiesta también en la autoría de los propios artículos. Resulta bastante complejo hacer una síntesis en este sentido, ya que hay una gran dispersión en cuanto a nombres. Los que aparecen con mayor frecuencia son Baylina, Garcia Ramon y Ortiz, todas ellas miembros de la Universidad Autónoma de Barcelona (UAB); junto con Domingo, de la Universidad de Valencia, y Sabaté, de la Complutense de Madrid. Los hombres tienen una presencia reducida, que se agranda al considerar la masculinización de nuestra disciplina. El informe sobre la investigación geográfica en España (1990-2012) indica, en el capítulo elaborado por Sánchez Aguilera (2013), que, en 2012, las mujeres representaban solo el 33,79\% del personal docente e investigador (PDI), cuatro puntos menos que en $1992^{14}$. En este contexto

14. La (re)masculinización de la geografía académica española ya fue puesta de manifiesto reiteradamente en diferentes artículos, como Garcia Ramon y Pujol (2004), Pujol et al. (2009) y Pujol et al. (2012), entre otros. 
de desigualdad, llama la atención que únicamente el 11,7\% de los artículos están firmados por hombres, a lo que le añadimos otro 13,4\% en el que aparecen conjuntamente hombre y mujer. Es decir, los hombres, solos o no, son responsables de una cuarta parte del total de artículos. Entre ellos, destacan los profesores Feo, de la Autónoma de Madrid; Viruela, de la Universidad de Valencia, o Tulla, de la UAB.

En el ámbito de las universidades, la UAB tiene una gran relevancia, al acaparar el 28,5\% de los artículos. Muy lejos de este valor están las universidades de Valencia y la Complutense de Madrid, con el 11\% y el 8\%, respectivamente. En el lado opuesto, encontramos algunas universidades muy significativas en el panorama español, ya sea el geográfico o el universitario en general, que tienen una representación muy baja o incluso nula en algunas ocasiones. Es el caso de la Universidad de Barcelona, la Autónoma de Madrid o la UNED, entre otras ${ }^{15}$. Lo mismo se puede decir del CSIC, que aparece referenciado en solo dos autorías.

Analizar los ámbitos territoriales de la investigación derivada de las publicaciones en revistas es bastante complejo. De entrada, porque muchas veces no hay especificaciones en el título que nos permitan concretar. Además, encontramos una abundancia relativa de artículos teóricos en los que no existen referencias territoriales definidas. En cualquier caso, en nuestro intento por clasificar, hemos hecho una propuesta escalar, en la que el mayor protagonismo corresponde a los estudios de carácter internacional referidos a uno o a varios países, si bien es cierto que frecuentemente se centran en lugares concretos. En segundo lugar, están los trabajos de ámbito local, que van desde pueblos hasta ciudades, comarcas o provincias. Por último, situamos a las comunidades autónomas, con un número menor de artículos.

Analizar el contenido de los artículos es una tarea que resulta especialmente complicada, por el carácter transversal que tienen muchos de ellos. Como en los casos anteriores, el título fue el elemento central de la investigación, si bien en esta ocasión, con frecuencia, tuvimos que recurrir a los resúmenes o incluso a la lectura completa de los textos para poder definir bien el ámbito temático. Las múltiples posibilidades fueron reducidas a ocho ítems, en el que se incluyó uno con la denominación «Otros» que actúa como cajón de sastre. Al final, los grupos temáticos son muy parecidos a los que en su día propusieron, en un estudio similar a este, Garcia Ramon y Caballé (1998) e incluso los resultados que dan para los países de habla francesa y el Mediterráneo son bastante semejantes a los de la tabla 2, con la excepción del tema urbano, que aquí pierde peso en relación con el estudio de 1998.

A pesar de las dificultades existentes, hay tres características que queremos señalar. La primera es la importancia de las contribuciones teóricas y meto-

15. El hecho de que algunas universidades no dispongan de revistas propias puede haber sido un condicionante para entender los resultados. Ahora no es tan común, pero anteriormente había una marcada tendencia a facilitar que las revistas fuesen el órgano de difusión de la investigación de los departamentos universitarios. 
Tabla 2. Principales temas de estudio

\begin{tabular}{lc}
\hline Tema & Valores expresados en tantos por ciento \\
\hline Teoría y metodología & 15,6 \\
Demografía & 9,5 \\
Mercado laboral & 22,3 \\
Rural & 22,3 \\
Urbano & 12,8 \\
Educación & 3,6 \\
Medio ambiente & 5,6 \\
Otros & 8,4
\end{tabular}

Fuente: revistas de geografía españolas.

dológicas, tema relevante por su escasez en la geografía española. Un segundo rasgo se refiere al destacado papel de la temática rural y laboral. Ortega Valcárcel (2000) ya ponía el énfasis en la impronta de las relaciones entre género y mundo rural y agrario, pero también el mercado laboral tiene un protagonismo singular. Incluso hemos encontrado grandes dificultades para separar estos dos ítems; por ejemplo: casi la mitad de los artículos del apartado rural tienen como tema principal el laboral.

Un tercer elemento a destacar es la menor importancia de los estudios urbanos e incluso de los relacionados con la población, en donde se destaca el papel de las mujeres inmigrantes. En general, existe una preocupación por el papel de la mujer inmigrante desde diferentes perspectivas, como la demográfica o la laboral, ya sea en el ámbito urbano o sobre todo en el rural.

Destaca Ortega Valcárcel (2000) que es difícil hacer una valoración de la geografía del género, debido a que coexisten planteamientos teóricos y metodológicos muy dispersos: desde los que se ligan al pensamiento crítico contemporáneo bajo la influencia del feminismo y de otras corrientes, hasta otros posicionamientos que se limitan a visibilizar a la mujer desde un punto de vista descriptivo y continuista. En estos últimos casos, no resultó fácil incorporar ciertos artículos al campo de la geografía del género, ya que la mujer aparecía como una variable, a veces la principal, dentro de un análisis espacial aparentemente neutro. Es cierto que son una minoría los que responden a estas características, pero representan un importante aspecto sobre el que es necesario reflexionar.

La internacionalización que desde el principio mostró la geografía del género en España tiene dos caras. La primera se visibiliza en la destacada presencia de artículos en revistas cuya autoría está vinculada a personas que trabajan en centros extranjeros. Son el 30\% los escritos en los que, por lo menos, una de las firmas pertenece a investigadoras e investigadores de un centro de fuera del Estado. Sin embargo, es necesario hacer algunas matizaciones importantes en este sentido: son prácticamente tres las revistas que concentran estos trabajos. El 63\% de los mismos se publicaron en DAG. Con todo, es en Scripta Nova donde los dos tercios de los artículos sobre género tienen autoría internacional, 
el 58,3\% lo encontramos en Treballs y el 43\%, en DAG. Además, el perfil de las investigadoras y de los investigadores en cada una de estas tres revistas es diferente.

En Treballs, poco menos de la mitad de los artículos están firmados por Benería, autora catalana que trabaja en la Universidad de Cornell (EUA). En Scripta Nova, es América Latina, sobre todo Brasil, quien acapara todos los artículos con autoría extranjera. Por último, $D A G$ presenta un panorama más diversificado entre Europa, las Américas e incluso África. De hecho, el monográfico del número 49 (2007) lleva por título «Una mirada internacional a la geografía y al género», denominación bien significativa de ese carácter abierto y transfronterizo.

\subsection{La producción en revistas extranjeras}

La segunda cara de la internacionalización se expresa a través de la presencia de la geografía española en revistas foráneas. En este sentido, de las diecisiete publicaciones consultadas, el primer hecho que nos llama la atención es el escaso número de artículos a nivel general que hemos encontrado. Es cierto que esta afirmación tiene que ser matizada, ya que, independientemente de otras cuestiones, la selección que hemos hecho incluye algunas de las revistas más prestigiosas del panorama geográfico y que, además, evidencian una importante transdisciplinariedad. De hecho, algunas, como Sociologia Ruralis, no son estrictamente de geografía y, por lo que se refiere a otras, resulta complicado adscribirlas a una sola disciplina.

Es cierto que solo hemos encontrado dos revistas en las que no aparece ningún artículo de autoría española. En el lado opuesto, se sitúan otras como Geoforum, International Journal of Urban and Regional Research o Population, Space and Place. Con todo, es necesario hacer de nuevo un par de puntualizaciones. En primer lugar, esa representación española no siempre viene desde la geografía y, de hecho, en revistas como Environment and Planning $A$ o Tijdschrift voor Economische en Sociale Geografie (TESG), entre otras, un buen número de artículos son de economía o de sociología, por ejemplo. En segundo lugar, solo Rodríguez Pose, de la Economics School of London, aparece con frecuencia como autor en un número significativo de revistas. A continuación, situamos a Garcia Ramon, Saurí, Bayona, etc., para dar paso finalmente a otras personas con un número mucho más reducido de textos.

Centrándonos exclusivamente en los temas de género, hemos encontrado solo once artículos en las diecisiete publicaciones analizadas, sin ningún año que destaque especialmente. De ellos, seis tienen como autora o coautora a Garcia Ramon. En cierta medida, el resto de las (co)autorías corresponden prácticamente a las mismas personas que publican en España, como Baylina, Ortiz o Prats, todas de la UAB. Las excepciones están en Domínguez y Ávila (2013) y Gurrutxaga (2013), que no aparecen referenciados en la bibliografía española de geografía del género. Además, también hemos encontrado algunos textos cuya autoría proviene de otras disciplinas, aunque aborden la cuestión de 
género. En todo caso, su número es muy pequeño y no han sido considerados en este estudio.

Con todo, es necesario hacer alguna aclaración, puesto que, en la cifra de once artículos, se incluyen algunos que no son exactamente de investigación. Es el caso, por ejemplo, de Garcia Ramon y Caballé (1998), que publican un texto en TESG bajo el paraguas de "Estado del Arte», o el homenaje que Garcia Ramon y Luna (2007) hacen a J. Monk en Gender, Place and Culture. Pero, en el lado contrario, no podemos reducir la presencia de estas autoras a los artículos de investigación, puesto que también se manifiestan bajo otras formas, como book reviews, guest editorials o viewpoints. En este sentido, y por indicar solo algunas citas, estaría Prats, con un review essay en 2014 en Gender, Place and Culture; en la misma revista, Garcia Ramon et al. (2006), con un guest editorial; Prats (2002), con un book review en Progress in Human Geography, o Garcia Ramon y Baylina, también en 2002, informando sobre el congreso «El nuevo papel de las mujeres en el desarrollo rural», al que acompañan un par de textos breves de otras autoras en la prestigiosa Antipode.

En cuanto a las revistas en las que publican, se advierte una clara concentración en Gender, Place and Culture frente a otras de carácter más generalista. De hecho, en ella, aparecen cuatro de los artículos, además de otros textos menores bajo fórmulas como las citadas en el párrafo anterior. Además, Prats forma parte de su consejo editorial en el cuatrienio 2006-2009. El resto de los artículos aparecen dispersos. Destacan, en todo caso, Geoforum, con dos trabajos.

Para ir terminando, queremos hacer un breve comentario sobre el contenido de estos trabajos. Resulta complicado mantener la misma clasificación que hemos hecho para las revistas españolas, porque la temática marca diferencias significativas. El mundo rural sigue teniendo una cierta importancia con tres artículos. Con este mismo número, aparecen otros textos en los que se pone el acento en la propia academia, desde las aportaciones de Monk hasta la masculinización de la geografía o un estudio bibliométrico. Las otras cinco aportaciones resultan más difíciles de encasillar: dos representan una novedosa mirada a la geografía colonial desde una perspectiva feminista. En una línea con ciertas semejanzas, Domínguez y Ávila (2013) exploran la narrativa autobiográfica de tres inmigrantes de Japón en las Islas Canarias. Los dos últimos textos abren caminos apenas trillados en la geografía española relacionados con la infancia y la juventud.

$\mathrm{Ni}$ los artículos ni otros textos que hemos comentado llenan el depósito de la internacionalización, aunque es cierto que dejan poco espacio. Creemos que el panorama de la geografía del género y de las sexualidades en España no es mucho mayor del que hemos dibujado. La revisión de los curriculum vitae de las autoras más destacadas en las temáticas analizadas nos permite comprobar que, efectivamente, sus trabajos aparecen en otras revistas, no solo anglófonas, y también en formato libro. Sin embargo, nos estamos refiriendo a un número muy reducido de personas, eso sí, con gran dinamismo y, afortunadamente, con capacidad de renovación aún a pesar de las dificultades existentes. 


\section{Conclusiones}

A partir del vaciado de las revistas de geografía, hemos comprobado que el género y la sexualidad son temáticas muy secundarias en el ámbito español, sobre todo el segundo aspecto. Como hemos demostrado, a pesar de que se encuentran trabajos pioneros sobre género en fechas tan tempranas como la década de 1980, su despegue nunca llegó a acontecer, de manera muy clara en lo referido a la sexualidad y con matices en el género. Las afirmaciones de Ortega Valcárcel (2000) se confirman de forma parcial en cuanto a que los estudios de género son realizados fundamentalmente por mujeres y tienen un foco principal en el mundo rural, además de la fuerte carga teórica y metodológica. Otras características se resumen en el protagonismo de un reducido número de autoras, en el papel de $D A G$ y de la UAB. También nos parece relevante la presencia de autoras extranjeras y, al mismo tiempo, la internacionalización de un reducido número de investigadoras que no solo publican en revistas de referencia, sino que también, en algunos casos, muestran una significativa actividad con aportaciones menores.

Para concluir, nos parece pertinente tratar de explicar los motivos que, a nuestro juício, han derivado en la situación actual, que, para la sexualidad, y a la vista de los datos, se puede calificar de precariedad, y para el género, de mejorable. Para ello, expondremos cinco argumentos que será necesario desarrollar en trabajos futuros.

1. En general, son consideradas temáticas poco relevantes y fuera del discurso político dominante. A pesar de las evidencias sobre las desigualdades de género, se ha producido una absorción por parte del sistema que se manifiesta, por ejemplo, en organismos que tienen como misión velar por la igualdad. Cualquier debate fuera de ese contexto institucional que se permea hacia la sociedad es calificado de trasnochado, como en cierta medida es considerado el propio feminismo. Por lo que se refiere a la sexualidad, probablemente existan miedos personales a una identificación con la orientación sexual estudiada, y el caso de Valentine (1998) es claro, paradójicamente, a pesar de los avances sociales en la aceptación del colectivo LGTB (lesbianas, gays, personas transgénero y bisexuales).

2. A pesar de los profundos cambios experimentados, la geografía en España, todavía en la actualidad, arrastra algunas inercias de los años de la dictadura. Además, ahora, existe una obsesión por la utilidad social de nuestra disciplina, que da, a nuestro entender, un valor excesivo a las herramientas tecnológicas frente al debate teórico y a los problemas socioterritoriales. Aquel, casi siempre ausente, margina una temática que parte como una alternativa teórica y epistemológica (Ortega Valcárcel, 2000).

3. El sistema académico español actual no favorece el desarrollo de estas geografías. Por una parte, los proyectos de investigación de convocatorias competitivas ${ }^{16}$ no dan preferencia a estas temáticas, como se desprende de los datos

16. Muchas veces, estos proyectos son la base para elaborar artículos de revista. 
del Informe sobre la investigación geográfica en España (Lasanta y Martín, 2013), aunque también puede ser que no haya solicitudes potentes. Por otro lado, el modelo de sexenios, que está otorgando un gran peso a las revistas, sirve también para ejercer presión sobre aquellas mejor posicionadas que se han visto obligadas a incrementar su volumen. En este contexto, resulta más complicado incorporar artículos de temáticas secundarias.

4. En cierto modo, en relación con lo anterior, se observa que algunas autoras optan por publicar en revistas extranjeras, a fin de aprovechar no solo el mayor valor académico que se les atribuye, sino también la fuerte internacionalización que caracteriza a las personas que investigan en geografía del género. En todo caso, no es una tarea fácil por varios motivos: la exigencia y la competitividad; el esfuerzo que supone adaptarse a la diferente estructura mental que requieren estas publicaciones, o, simplemente, razones económicas derivadas de la necesidad de realizar traducciones muy precisas.

5. Por último, creemos que la crisis económica actual está bloqueando la entrada de gente joven a las universidades, por lo que la innovación se resiente de manera evidente. Hay menos dinero para becas, menos proyectos de investigación y, en definitiva, menos posibilidades para financiar la elaboración de una tesis doctoral. Este hecho impacta directamente en las publicaciones científicas, fundamentalmente en las revistas, en donde, como ya hemos dicho, la competencia por parte del profesorado funcionario para la obtención de sexenios es cada vez mayor. Esta situación resulta bastante lamentable, porque la geografía tiene mucho que aportar a los estudios de género y sexualidad. La variable espacial es importante para entender, por ejemplo, el uso diferencial de la ciudad por parte de estas personas, así como para sus estrategias de integración o de refuerzo identitario.

\section{Referencias bibliográficas}

Bowlby, Sophie; Foord, Jo y Mackenzie, S. (1982). «Feminism and Geography». Area, 14 (1), 19-25.

CÀnoves, Gemma (1999). «Introducció». Documents d'Anàlis Geogràfica, 35, 15-18.

CÀnoves, Gemma y Solsona, Montserrat (1989). «En torno al seminario sobre "Agricultura género y espacio"”. Documents d'Anàlisi Geogràfica, 14, 213-217.

CASTELls, Manuel (1983). The city and the grassroots. Londres: Edward Arnold.

Corral, José y CÀnoves, Gemma (2013). «La investigación turística publicada en revistas turísticas y no turísticas: Análisis bibliométrico de la producción de las universidades catalanas». Cuadernos de Turismo, 31, 55-81.

Domínguez, Josefina y Ávila, Rosalía (2013). «The in-between lives of Japanese inmigrants in Las Palmas de Gran Canaria, Spain». Gender, Place and Culture [en línea], 20 (7), 896-913. <http://dx.doi.org/10.1080/0966369X.2012.737767>

Dóniz, Francisco Javier (2013). «El ocio gay masculino en Tenerife (Canarias, España): ¿Potencial producto turístico para la isla?». Revista de Estudios Turísticos, 197, 135-148. 
Esparcia, Javier (2013). «La investigación en geografía en los planes nacionales: Elemento de consolidación de la disciplina». En: Lasanta, Teodoro y Martín Vide, Javier (coords.). La investigación geográfica en España (1990-2012). Madrid: CSIC, 87-117.

EtTorre, E. M. (1978). "Women, urban social movements and the lesbian ghetto». International Journal of Urban and Regional Research [en línea], 2, 499-520. <http://dx.doi.org/10.1111/j.1468-2427.1978.tb00764.x>

García Ballesteros, Aurora (1982). «El papel de la mujer en el desarrollo de la geografía». En: Durán, María Ángeles. Liberación y utopía. Madrid: Akal Universitaria, 119-141.

Garcia Ramon, Maria Dolors (1985a). «El análisis de género y la geografía: Reflexiones en torno a un libro reciente». Documents d'Anàlisi Geogràfica, 6, 133-143.

- (1985b). Teoría y método en la geografía humana anglosajona. Barcelona: Ariel.

- (1989). «Género, espacio y entorno: ¿Hacia una renovación conceptual de la geografía? Una introducción». Documents d'Anàlisi Geogràfica, 14, 7-13.

Garcia Ramon, Maria Dolors y Baylina, Mireia (2002). "Comment on El nuevo papel de las mujeres en el desarrollo rural». Antipode [en línea], 34 (5), 1011-1013. <http://dx.doi.org/10.1111/1467-8330.00293>

Garcia Ramon, Maria Dolors y Caballé, Alba (1998). «Situating gender geographies: A bibliometric analysis». TESG (Journal of Economic and Social Geography), 89 (2), 210-216.

Garcia Ramon, Maria Dolors y Cànoves, Gemma (1988). "The role of women on the family farm: The case of Catalonia». Sociologia Ruralis [en línea], 28 (4), 263-270. <http://dx.doi.org/10.1111/j.1467-9523.1988.tb00344.x>

Garcia Ramon, Maria Dolors; Castañer, Margarida y Centelles, Núria (1988). "Women and geography in Spanish universities». The Professional Geographer [en línea], 40 (3), 307-315. <http://dx.doi.org/10.1111/j.0033-0124.1988.00307.x>

Garcia Ramon, Maria Dolors y Luna García, Tony (2007). «Challenging Hegemonies through Connecting Places, People and Ideas: Jan Monk's contribution to international gender geography». Gender, Place and Culture [en línea], 14 (1), $35-41$. <http://dx.doi.org/10.1080/09663690601122200>

Garcia Ramon, Maria Dolors y Pujol, Herminia (2004). «La presencia de las mujeres en la geografía académica: ¿Hacia una masculinización de la disciplina?». Cuadernos de Geografía, 75, 91-101.

Garcia Ramon, Maria Dolors; Simonsen, Kirsten y Vaiou, Dina (2006). «Guest Editorial: Does Anglophone hegemony permeate Gender, Place and Culture?». Gender, Place and Culture [en línea], 13 (1), 1-5. <http://dx.doi.org/10.1080/09663690500530867>

Gurrutxaga, Mikel (2013). "Changes in rural-urban sex ratio differences in the young professional age group as an indicator of social sustainability in rural areas: A case study in continental Spain, 2000-2010». Area [en línea], 45 (3), 337-347. <http://dx.doi.org/10.1111/area.12024>

KNOPP, Lawrence (1990). «Social consequences of homosexuality». Geographical Magazine (mayo), 20-25.

Lasanta, Teodoro y Martín Vide, Javier (2013). La investigación geográfica en España (1990-2012) [en línea]. Madrid: CSIC. <http://www.age-geografia.es/site/wpcontent/uploads/2015/06/Investigacion_Geogr.pdf>. 
LAURIA, Michael y KnOpP, Lawrence (1985). «Toward an analysis of the role of the gay communities in the urban reinassance». Urban Geography [en línea], 6, 152-169. <http://dx.doi.org/10.2747/0272-3638.6.2.152>

Martín Lou, María Asunción; Bodega Fernández, María Isabel; Cebrián de Miguel, Juan Miguel y Jiménez Royo, Ana (2013). «Las publicaciones de geógrafos españoles en revistas de geografía españolas». En: LAsAnta, Teodoro y MarTín VIDE, Javier (coords.). La investigación geográfica en España (1990-2012). Madrid: CSIC, 235-248.

Monk, Janice y Hanson, Susan (1989). «Temas de geografía feminista contemporánea». Documents d'Anàlisi Geogràfica, 14, 31-50.

Nogué, Joan y Romero, Joan (eds.) (2006). Las otras geografias. Valencia: Tirant Lo Blanch.

Olcina Cantos, Jorge y Lois González, Rubén Camilo (2013). «Grupos y redes de investigación en la geografía española: De la investigación individual a la investigación multidisciplinar». En: Lasanta, Teodoro y Martín Vide, Javier (coords.). La investigación geográfica en España (1990-2012). Madrid: CSIC, 119-146.

Ortega Valcárcel, José (2000). Los horizontes de la geografía. Barcelona: Ariel.

Palm, Risa y Pred, Allan (1976). A time-geographic approach on problems of inequality for women. Berkeley: University of California. Institute of Urban and Regional Development. Working Paper, 26.

Prat, José María (2014). «Nuevos planteamientos y nuevas motivaciones en la elección de destinos de turismo cultural-urbano homosexual: El Festival Circuit de Barcelona». Cuadernos de Turismo, 33, 311-334.

Prats, Maria (2002). «Book Review: El nuevo papel de las mujeres en el desarrollo rural». Progress in Human Geography, 26 (6), 840-842.

- (2014). «Review Essay». Gender, Place and Culture [en línea], 21 (2), 262-265. <http://dx.doi.org/10.1080/0966369X.2014.883732>

Pujol, Hermínia; Garcia Ramon, M. Dolors y Ortiz, Anna (2012). «El profesorado universitario de Geografía en España y sus trayectorias profesionales: Una mirada de género». Boletín de la AGE, 59, 323-344.

Pujol, Hermínia; Ortiz, Anna y Garcia Ramon, M. Dolors (2009). «La presencia y la carrera profesional de las mujeres en la geografía académica: Estudio de las peculiaridades del caso español». Estudios Socioterritoriales: Revista de Geografía, 7, 136-159.

Rodó, Maria (2013). "Young lesbians negociating public space: An intersectional approach through places». Children's Geographies [en línea].

- (2014). «Developing geographies of intersectionality with Relief Maps: Reflections from youth research in Manresa, Catalonia» Gender, Place and Culture, 21 (8), 925-944.

<http://dx.doi.org/10.1080/0966369X.2013.817974>

Sabaté Martínez, Ana (1984a). «Mujer, geografía y feminismo». Anales de Geografía de la Universidad Complutense, 4, 37-53.

- (1984b). «La mujer en la investigación geográfica». Anales de Geografía de la Universidad Complutense, 4, 275-282.

SÁnchez Aguilera, Dolores (2013). «La investigación y los investigadores en Geografía en cifras. Una aproximación». En: LAsanta, Teodoro y Martín Vide, Javier (coords.). La investigación geográfica en España (1990-2012). Madrid: CSIC, 43-58. 
Santos, Xosé (1996). "Xénero, Comunidades e Espacio». En: Rodríguez Casal, Antón (coord.). Humanitas: Estudios en homenaxe ó prof. Dr. Carlos Alonso del Real. Volumen 2. Santiago: Universidad de Santiago de Compostela, 743-762.

- (1998). «O espacio da homosexualidade en Galicia». Fluxos, 1, 23-34.

- (2006). «Territorio e identidad: Sexualidades y estrategias espaciales». En: Buxán Bran, Xosé (ed.). Lecciones de disidencia: Ensayos de critica homosexual. Madrid: Egales, 45-60.

Sanz Casado, Elías y Martín Moreno, Carmen (1997). «Técnicas bibliométricas aplicadas a los estudios de usuarios». Revista General de Información y Documentación, 8 (2), 41-68.

Silva San Román, Lily Dorothy (2006). Producción y uso de espacios lésbicos en Salamanca. Trabajo de investigación tutelado por Teresa Vicente Mosquete. Universidad de Salamanca, 65.

Valentine, G. (1998). "Sticks and stones may break my bones”: A personal geography of harassment». Antipode [en línea], 30 (4), 305-332. <http://dx.doi.org/10.1111/1467-8330.00082>

Zelinsky, Wilbur (1973). "Women in Geography: A brief factual account». The Professional Geographer [en línea], 25, 101-106.

<http://dx.doi.org/10.1111/j.0033-0124.1973.00101.x> 